\title{
Propuesta de innovación docente en arquitectura mediante el aprendizaje basado en problemas
}

\section{University teaching innovation proposal in architecture by means of problem-based learning}

María Victoria Requena García de La CRUZ

ORCID: https://orcid.org/0000-0002-2410-706X

Universidad de Sevilla

Departamento de estructuras de

edificación e ingeniería del terreno

mrequena1@us.es

Fecha de recepción: 19-05-2019

Fecha de aceptación: 11-06-2019

DOI: http://dx.doi.org/10.12795/9788447221912.019

Pp.: 449-468 
Este trabajo presenta una propuesta de innovación docente en la asignatura de Mecánica del Suelo y Cimentaciones, del Grado en Fundamentos de la Arquitectura. Se trata de una asignatura técnica con alto nivel de dificultad y densidad de conceptos. Por ello y para mejorar el aprendizaje del alumnado, la experimentación docente resulta necesaria. Esta ha consistido en la realización de un Ciclo de Mejora en el Aula (CIMA) en dos sesiones, de cuatro y dos horas de duración cada una, aplicado al tema: reconocimiento del terreno. El ciclo se basa en el modelo didáctico alternativo denominado "Aprendizaje basado en problemas" y breves aportaciones de la profesora. Para ello, se ha realizado una secuencia de actividades, siendo la última la de mayor importancia y duración, centrada en la resolución de un problema real. Los resultados de este ciclo de mejora han sido satisfactorios. Los alumnos asimilaron la sucesión de actividades y mejoraron su aprendizaje.

Palabras clave: Mecánica del suelo y cimentaciones; Grado en Fundamentos de la Arquitectura; Docencia universitaria; Experimentación docente universitaria; Aprendizaje basado en problemas.

\section{Abstract}

This paper presents a teaching innovation proposal applied to the Soil Mechanics and Foundations subject, in the Degree in Architecture Fundamentals. This is a very technical subject of high level of difficulty and density of concepts. Therefore, in order to improve the students' learning, the experimentation is necessary. This experimentation has consisted in an Improvement Cycle in Classroom (ICIC) applied to the "soil recognition" topic during two sessions of four and two hours of duration, respectively. The cycle is based on the alternative didactic model, which is focused on the problem-based learning with brief contributions by the teacher. A sequence of activities has been developed, being the last activity the most important one based on the resolution of a real problem. The results of this cycle have been satisfactory. The students have assimilated the sequences and improved their learning.

Keywords: Soil Mechanics and Foundations; Degree in Architecture Fundamentals; University teaching; University teaching experimentation; Problem-based learning. 


\section{Breve descripción del contexto}

La asignatura de Mecánica del Suelo y Cimentaciones (MSC) se imparte en el segundo cuatrimestre del tercer curso del grado en Fundamentos de la Arquitectura (Plan 2012) en la Escuela Técnica Superior de Arquitectura de Sevilla. Se trata de una asignatura muy técnica con un alto nivel de dificultad y densidad de conceptos. Además, es la primera vez que los estudiantes se enfrentan a la complejidad que supone el estudio del comportamiento del suelo. Pese a tener una estrecha relación con las asignaturas de Estructuras que se imparten durante el grado, estas no profundizan en el estudio de la cimentación y del comportamiento del suelo. Por ello, el nivel de conocimiento de los estudiantes antes de cursar la asignatura es relativamente básico. En líneas generales, la asignatura se centra en las siguientes cuestiones:

- Identificación de tipos de suelos, tipologías de cimentaciones, estructuras de contención, sistemas de mejora y recalce y excavaciones.

- Diseño, cálculo, análisis y definición de estructuras de suelo, cimentaciones, estructuras de contención, patología y recalce.

- Seguridad en las cimentaciones según normativa.

- Estudio y análisis del comportamiento del suelo.

Dada la complejidad de la asignatura, la experimentación docente resulta necesaria para mejorar el aprendizaje de los alumnos. Por ello, en este trabajo se presenta una propuesta de innovación docente realizada durante las sesiones séptima y octava del curso académico 20182019. La duración del ciclo ha sido de cuatro y dos horas durante la primera y la segunda sesión del ciclo respectivamente. Esta experimentación ha consistido en la realización de un Ciclo de Mejora en el Aula (CIMA) aplicado al tema "Reconocimiento del terreno". Este tema fue seleccionado por englobar la mayor parte de los contenidos conceptuales de la asignatura. 
El ciclo se divide en tres partes: una parte práctica realizada en el laboratorio; una breve introducción teórica combinada con una actividad; y, por último, una actividad práctica principal y de mayor duración. Esta última se ha planteado según el método de aprendizaje basado en problemas.

El número de alumnos en la clase es reducido, 24 en total. Para la realización de las actividades, los alumnos se dividen en grupos de tres o cuatro integrantes. El aula donde se imparte la asignatura cuenta con sillas y mesas no fijas para permitir y fomentar el trabajo en grupo.

\section{Diseño previo del Ciclo de Mejora en el Aula}

El objetivo de este CIMA es acercar al alumno a la problemática real a la que tendrá que hacer frente durante su vida profesional: la elaboración de una campaña de reconocimiento del terreno y el análisis de la información contenida en un estudio geotécnico. Para ello, el alumno debe ser capaz de interpretar y aplicar la normativa al respecto.

\section{Mapa de contenidos}

Los contenidos del diseño del ciclo de mejora son de tipo conceptual, procedimental y actitudinal. Todos se han considerado para la previsión de las actividades realizadas en las dos sesiones del ciclo. Los contenidos se organizan en forma de mapa (Figura 1) y son los siguientes:

\section{Conceptuales:}

- Descripción y análisis de un estudio geotécnico.

- Identificación de la información necesaria que debe incorporar un estudio geotécnico.

- Descripción de una campaña de reconocimiento de un terreno. 
- Distinción entre reconocimiento profundo y superficial de un terreno.

- Distinción entre ensayos in situ, de laboratorio, sondeo y tomamuestras.

Procedimentales:

- Elaboración del índice de la documentación contenida en un estudio geotécnico.

- Manejo, análisis e interpretación de normativa.

- Aplicación de la normativa para la determinación de la campaña de reconocimiento.

- Simulación y elaboración de una campaña de reconocimiento para un caso real.

- Observación de la realización de los ensayos in situ y de laboratorio.

Actitudinales:

- Trabajo en equipo. El alumno aprende a respetar a sus compañeros y compañeras, respeta el turno de palabra, atiende a las opiniones de los compañeros y las valora.

- Esfuerzo en la toma de decisiones.

- Toma de consciencia de la responsabilidad del arquitecto en la elaboración del documento y en la determinación de la solución de cimentación de la obra.

- Interiorización de la necesidad de colaboración del arquitecto con la empresa a la que se encarga la realización del estudio.

En el mapa, se resaltan los contenidos vistos en otros temas y los conceptuales de primer y segundo nivel. Estos últimos incorporan contenidos procedimentales, ya que tratan, en mayor medida, del proceso de realización de los ensayos.

Jornadas de Formación e Innovación Docente del Profesorado | № 2 (2019) Esta obra se distribuye con la licencia Creative Commons Reconocimiento-NoComercial-SinObraDerivada 
María Victoria REQUENA GaRcía de LA CRUZ

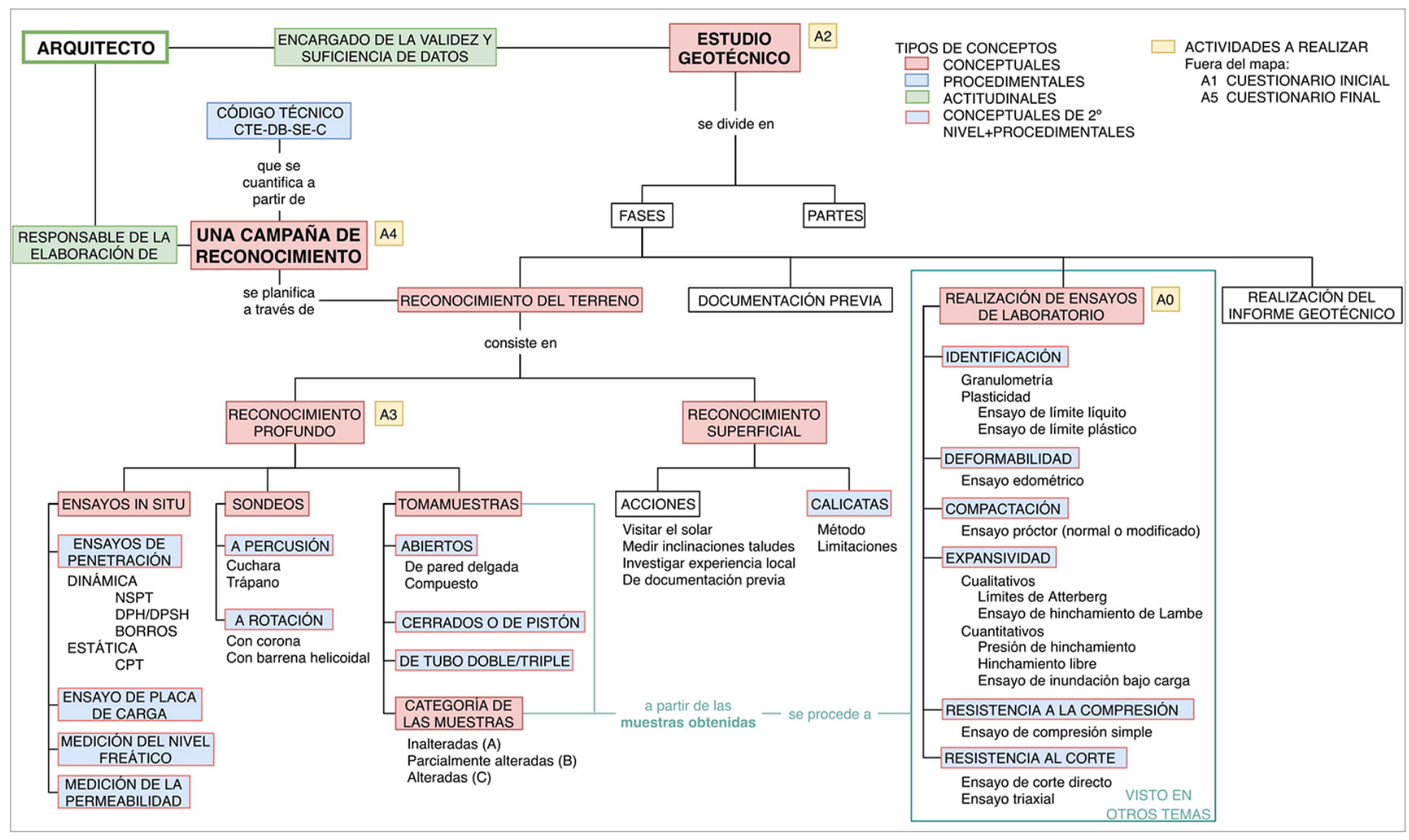

Figura 1.jpg

Jornadas de Formación e Innovación Docente del Profesorado I № 2 (2019)

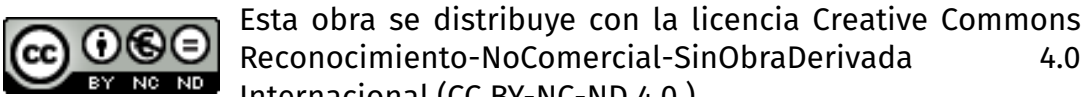

Internacional (CC BY-NC-ND 4.0.) 


\section{Modelo metodológico posible}

El modelo metodológico propuesto para este CIMA se centra en los estudiantes. Concretamente, se basa en el método de Aprendizaje Basado en Problemas (ABP) (Justo Moscardó, Delgado Trujillo, Vázquez Boza y Branda, 2016) y en el propuesto por D. Finkel (2008). El primer método implica un aprendizaje activo y cooperativo. Mediante la resolución de problemas, los alumnos deben ser capaces de buscar las herramientas teóricas necesarias para encontrar la solución. Este modelo favorece el trabajo autónomo y en grupo de los alumnos. Además, fortalece el compromiso y responsabilidad de los alumnos para con el grupo, que son dos de las cualidades más necesarias para desempeñar la profesión de arquitecto. Asimismo, el modelo propuesto por Finkel presenta los denominados "talleres conceptuales". Durante estos talleres, los alumnos también trabajan en la resolución de un problema basado en un caso real a partir de las breves aportaciones y orientaciones del profesor.

Para la organización del CIMA, se ha realizado una secuencia de actividades basada en los contenidos establecidos en el tema. Para ello, se han considerado las indicaciones de De Alba y Porlán (2017). La secuencia de actividades se muestra con más detalle en el apartado correspondiente a la aplicación del ciclo. Esta se divide en seis actividades, siendo cuatro las principales y dos auxiliares. Las cuatro principales se han planteado acorde con el aprendizaje activo de los estudiantes. Las otras dos actividades se realizan para evaluar el aprendizaje de los alumnos antes y después de la aplicación del ciclo de mejora

El modelo metodológico general se basa -como se ha dicho- en el ABP y en los "talleres conceptuales". Sin embargo, se desarrolla una experiencia, que es la actividad principal del ciclo, más específica y de aplicación práctica 
del método. En la primera actividad, los alumnos deberán realizar los ensayos de identificación del suelo según una breve explicación técnica. La segunda y tercera actividad se basan en una breve introducción teórica por parte del profesor y en el trabajo de los alumnos con un documento básico para su actividad profesional futura. La tercera actividad se centra en un problema basado en un caso real. Para resolverlo, los alumnos deben buscar las herramientas teóricas necesarias trabajando en grupos, donde debaten y ponen en común lo aprendido por cada uno individualmente. Al igual que en el modelo de Finkel (2008), el profesor se limita a conducirlos participando puntualmente en los grupos mientras debaten. Al final, los alumnos deben entregar un documento con la resolución del problema planteado.

\section{Cuestionario inicial y final}

Para valorar el estado inicial y final del conocimiento de los estudiantes se diseñan y aplican unos cuestionarios. Ambos incluyen las mismas preguntas para poder valorar realmente cómo ha sido el aprendizaje de los alumnos en relación con las cuestiones exploradas. Estas cuestiones engloban los cinco contenidos conceptuales de primer nivel del tema: definición de un estudio geotécnico, de una campaña de reconocimiento y de los tipos de reconocimiento del terreno (profundo, superficial o a través de ensayos de laboratorio). Al terminar la sesión, los alumnos deben ser capaces de responder adecuadamente a las cinco cuestiones planteadas.

La evaluación de los cuestionarios se realiza a modo de escalera de aprendizaje siguiendo las indicaciones establecidas en Rivero y Porlán (2017). Para ello, se han elaborado 4 escalones que representan una gradación de menor (escalón 0) a mayor nivel (escalón 3) de conocimiento. El nivel 0 de conocimiento agrupa respuestas erróneas, en 
blanco o sin sentido. El nivel 1 recoge respuestas que demuestran un nivel de conocimiento muy básico sin argumentación. En un principio, estos escalones se plantearon como uno solo. Sin embargo, tras el análisis de las respuestas de los alumnos, surgió la necesidad de crear un nivel de conocimiento 0 . El escalón 2 engloba respuestas correctas que incluyen una argumentación muy básica. El escalón 3 recoge respuestas que demuestran conocimiento alto y argumentado sobre la cuestión. Las preguntas que se han incluido en ambos cuestionarios, así como los tipos de respuesta, se muestran en la Tabla 1.

Tabla 1. Preguntas, escalones y grupos de respuestas incluidos en los cuestionarios.

\begin{tabular}{|c|c|}
\hline \multicolumn{2}{|c|}{ P1. ¿En qué consiste un estudio geotécnico? } \\
\hline Escalón 0 & $\begin{array}{l}\text { No responde a la pregunta o responde erróneamente al } \\
\text { azar. }\end{array}$ \\
\hline Escalón 1 & Define vagamente en qué consiste un estudio geotécnico. \\
\hline Escalón 2 & $\begin{array}{l}\text { Conoce y describe de forma muy sencilla en qué consiste } \\
\text { un estudio geotécnico. }\end{array}$ \\
\hline Escalón 3 & $\begin{array}{l}\text { Conoce y describe de forma clara y concisa en qué consiste } \\
\text { un estudio geotécnico. }\end{array}$ \\
\hline \multicolumn{2}{|c|}{ P2. ¿Sabrías realizar una campaña de reconocimiento del terreno? } \\
\hline Escalón 0 & $\begin{array}{l}\text { No responde a la pregunta o responde erróneamente al } \\
\text { azar. }\end{array}$ \\
\hline Escalón 1 & $\begin{array}{l}\text { Conoce vagamente el proceso a seguir para realizar una } \\
\text { campaña de reconocimiento del terreno. }\end{array}$ \\
\hline Escalón 2 & $\begin{array}{l}\text { Tiene algunas nociones sobre cómo se debe realizar una } \\
\text { campaña de reconocimiento del terreno y define el proceso } \\
\text { de forma sencilla. }\end{array}$ \\
\hline Escalón 3 & $\begin{array}{l}\text { Conoce cómo se debe realizar una campaña de reconoci- } \\
\text { miento del terreno y define el proceso de forma adecuada. }\end{array}$ \\
\hline \multicolumn{2}{|c|}{$\begin{array}{l}\text { P3. ¿Qué responsabilidad tiene el arquitecto en una campaña de reco- } \\
\text { nocimiento del terreno y un estudio geotécnico? }\end{array}$} \\
\hline
\end{tabular}

Jornadas de Formación e Innovación Docente del Profesorado | № 2 (2019) Esta obra se distribuye con la licencia Creative Commons Reconocimiento-NoComercial-SinObraDerivada Internacional (CC BY-NC-ND 4.0.) 


\begin{tabular}{|l|l|}
\hline Escalón 0 & $\begin{array}{l}\text { No responde a la pregunta o responde erróneamente al } \\
\text { azar. }\end{array}$ \\
\hline Escalón 1 & Reconoce vagamente el papel o función del arquitecto. \\
\hline Escalón 2 & $\begin{array}{l}\text { Indica que el arquitecto tiene responsabilidad en la realiza- } \\
\text { ción de una campaña de reconocimiento y un estudio geo- } \\
\text { técnico, pero no argumenta la respuesta. }\end{array}$ \\
\hline Escalón 3 & $\begin{array}{l}\text { Indica que el arquitecto tiene responsabilidad en la realiza- } \\
\text { ción de una campaña de reconocimiento y un estudio geo- } \\
\text { técnico y, además, argumenta la respuesta. }\end{array}$ \\
\hline $\begin{array}{l}\text { P4. ¿En qué consiste el reconocimiento profundo y superficial del } \\
\text { terreno? }\end{array}$ & $\begin{array}{l}\text { No responde a la pregunta o responde erróneamente al } \\
\text { azar. }\end{array}$ \\
\hline Escalón 0 \\
\hline Escalón 1 & Identifica vagamente las cuestiones preguntadas. \\
\hline Escalón 2 & $\begin{array}{l}\text { Reconoce alguna diferencia simple sobre las cuestiones, } \\
\text { pero no las describe. }\end{array}$ \\
\hline Escalón 3 & Reconoce todas las diferencias y las puede describir. \\
\hline tras y ensayos de laboratorio?
\end{tabular}

\section{Aplicación del Ciclo de Mejora en el Aula}

En esta sección se muestra en primer lugar, la secuencia de actividades programada incluyendo un relato resumido del desarrollo real de las sesiones y, en segundo lugar, el proceso considerado para la evaluación del aprendizaje de los estudiantes.

Jornadas de Formación e Innovación Docente del Profesorado | № 2 (2019) Esta obra se distribuye con la licencia Creative Commons Reconocimiento-NoComercial-SinObraDerivada Internacional (CC BY-NC-ND 4.0.) 


\section{Secuencia de actividades programadas}

La secuencia programada para la aplicación del ciclo se divide en seis actividades: seis son principales y dos auxiliares. El modelo metodológico general se basa en el aprendizaje activo de los estudiantes, que ha sido considerado para el diseño de las actividades A0, A2, A3 y A4. Esta última actividad (A4) es además una experiencia más específica y de aplicación de los "talleres conceptuales" de Finkel (2008). Las actividades A1 y A5 sirven para valorar el nivel de aprendizaje antes y después de la aplicación del ciclo de mejora.

\section{A0. Visita al laboratorio}

Para esta actividad, los alumnos son recibidos en el laboratorio del área de Ingeniería del Terreno de la Escuela Técnica Superior de Arquitectura.

- Tiempo previsto: 120 minutos, en la primera sesión.

- Descripción: Con esta actividad, se pretende que los alumnos tengan un primer acercamiento a la realidad del laboratorio. Para ello llevan a cabo los ensayos junto a los técnicos. En primer lugar, la profesora realiza una breve descripción sobre las distintas propiedades de los suelos, para lo que se dispone de muestras, 22 en total. Al mismo tiempo, los alumnos van pasando por las muestras para observarlas, tocarlas e incluso olerlas. Pueden añadir agua para comprobar la permeabilidad o la cohesión de cada muestra. El objetivo es que sean capaces de identificar a simple vista los distintos tipos de suelos. Posteriormente, se les muestran cajas de sondeos, así como el proceso de preparación de las muestras para los distintos ensayos. A continuación, se realiza el ensayo de próctor normal, que analiza la compactación de un suelo, y el de compresión simple hasta la rotura de la probeta. Seguidamente, se llevan a cabo los ensayos de límite líquido y plástico y se les muestra el aparato de corte directo. Este último ensayo no se lleva a cabo debido 
al tiempo que sería necesario para su realización. A continuación, los alumnos realizan un ensayo de granulometría y se les muestran los aparatos de ensayo edométrico, triaxial y permeámetro. Para estos tres ensayos ocurre lo mismo que para el de corte directo.

- Contenidos: Conceptuales, los vistos desde el inicio de la asignatura; procedimentales, manejo de la normativa y realización de ensayos; actitudinales, interiorización de la necesidad de colaboración del arquitecto con la empresa a la que se encarga el estudio. Además, en esta actividad se emplean contenidos que se han ido viendo a lo largo de la asignatura.

- Desarrollo real: El clima era muy positivo dado que se trata de una actividad muy distinta de las habituales y que además se realiza fuera de la clase. La actitud de los alumnos reflejaba un nivel de interés mucho mayor a juzgar por la cantidad de preguntas que realizaban. Una dificultad fue la falta de tiempo para finalizar la actividad, empleándose unos 10 minutos más de lo previsto.

\section{A1. Cuestionario inicial}

A partir de esta actividad, todas se realizan en el aula donde normalmente se imparte la docencia.

- Tiempo previsto: 30 minutos, en la primera sesión.

- Descripción: Esta actividad se lleva a cabo para determinar el nivel de conocimiento de los alumnos previo a la realización del CIMA. Pese a que se trata de un cuestionario inicial, la actividad se realiza tras la visita al laboratorio. Durante la visita, los alumnos recuerdan lo aprendido en los temas anteriores y eso les ayuda a comprenderlos mejor. Hasta este momento, se les ha incidido en que, para la realización de los ensayos, se necesitan muestras, pero no se les ha dicho de dónde se obtienen y para qué. Por tanto, es posible realizar el cuestionario inicial justo a continuación de la visita.

- Contenidos: Todos los conceptuales incluidos en el mapa. 
- Desarrollo real: Durante la actividad, el ambiente era bastante distendido. Una de las dificultades analizadas fue que no se la tomaran en serio y que no respondieran sinceramente. Sin embargo, la actuación del alumnado fue más que satisfactoria. Se les explicó que se trataba de una actividad para mejorar la docencia de la asignatura.

A2. Ensayos in situ, sondeos y tomamuestras. Reconocimiento profundo vs. superficial del terreno

- Tiempo previsto: 45 minutos, en la primera sesión.

- Descripción: El objetivo de esta actividad es que los alumnos aprendan a diferenciar entre reconocimiento superficial y profundo. Para ello, se ha enfocado como una clase teórica breve centrada en los distintos tipos de ensayos in situ, sondeos y tomamuestras. Se emplean diapositivas y vídeos sobre cómo se realiza cada ensayo. Esta breve actividad es necesaria para llevar a cabo la siguiente actividad.

- Contenidos: Conceptuales, distinción entre reconocimiento profundo y superficial de un terreno y distinción entre ensayos in situ, de laboratorio, sondeo y tomamuestras; procedimentales, observación de la realización de los ensayos; actitudinales, toma de decisiones y consciencia de la responsabilidad del arquitecto en la determinación de la solución de cimentación del edificio.

- Desarrollo real: Al disminuir la carga teórica y utilizar nuevas herramientas, los alumnos parecían más interesados. También porque se les estaba explicando algunas preguntas incluidas en el cuestionario inicial. Supuso todo un avance dado que la breve aportación teórica se centró en lo importante del tema.

\section{A3. Estudio geotécnico}

- Tiempo previsto: 30 minutos, en la primera sesión.

- Descripción: Se pretende que los alumnos trabajen con un documento necesario para la elaboración de un proyecto de arquitectura. En pdf se les entrega un estudio geotécnico que deben analizar simulando un 
trabajo que tendrán que realizar durante su actividad profesional. A partir del análisis, deben determinar las partes en las que se divide, cómo se realiza y los ensayos que incluye. La profesora, a partir de la aportación de los estudiantes, crea el índice del documento en la pizarra y concluye destacando la importancia del documento.

- Contenidos: Conceptuales, descripción y análisis del estudio geotécnico e identificación de la información que debe incorporar; procedimentales, elaboración del indice del estudio geotécnico; actitudinales, trabajo en equipo, respeto a los compañeros y del turno de palabra, atender a las opiniones de los compañeros y valorar y tomar consciencia de la responsabilidad del arquitecto en la elaboración del documento.

- Desarrollo real: Durante la actividad, se consiguió que los alumnos participaran activamente, incluso aquellos que normalmente no lo hacen en el resto de clases. No se perdió tiempo por problemas informáticos, que era uno de los riesgos previstos de la actividad.

A4. Realización de una campaña de reconocimiento de un terreno

- Tiempo previsto: 15 minutos en la primera sesión y 90 minutos en la segunda.

- Descripción: Esta actividad se ha concebido como problema real sobre el que trabajar como una pequeña experiencia de aprendizaje basado en problemas. Los alumnos por grupos deberán enfrentarse a un problema que los arquitectos encuentran en cualquier proyecto. La actividad consiste en realizar una campaña de reconocimiento del terreno para un edificio real (ya construido). Durante los primeros 15 minutos de la primera sesión, se les reparte el enunciado y se dividen en grupos de trabajo. Los grupos son de tres a cuatro integrantes y se les asigna un número identificativo correspondiente al edificio sobre el que tendrán que hacer el trabajo. Durante esta primera sesión, la profesora comenta brevemente el enunciado. Los apartados que deberán responder son: 
- Planimetría y fotografias del edificio. Se debe incluir la superficie de ocupación, así como la clasificación de la edificación y del tipo del terreno según el código.

- Determinación, distribución y justificación de los puntos de reconocimiento a realizar.

- Determinación, distribución y justificación de los tipos de ensayos de penetración y sondeos a realizar.

- Determinación y justificación del corte geotécnico del terreno previsto según el documento ITE Sevilla.

- Determinación, explicación del proceso y justificación de los tipos de ensayos de laboratorio a realizar (para cada estrato).

- Determinación y justificación de los resultados de los ensayos obtenidos según el documento ITE Sevilla.

Previamente, como trabajo fuera de clase, cada integrante se encarga de resolver un apartado para convertirse en "experto". Durante la segunda sesión en clase, los alumnos trabajan en grupos resolviendo las cuestiones contándose lo que han estudiado y analizado cada uno por su cuenta. Para la realización de este trabajo, los alumnos deben emplear la normativa de aplicación, el CTE-DB-SE-C (Ministerio de Fomento de España, 2007). Como no se van a realizar los ensayos, los alumnos obtendrán esta información del Anejo I.2 del documento ITE Sevilla (Colegio de Arquitectos de Sevilla, 2010). Este documento recoge mapas y resultados de ensayos sobre las propiedades de los distintos estratos que normalmente se encuentran en Sevilla. La entrega de este trabajo se deberá realizar una semana después de esta sesión.

- Contenidos: Conceptuales, descripción de una campaña de reconocimiento de un terreno, distinción entre reconocimiento profundo y superficial de un terreno, y entre ensayos in situ, de laboratorio, sondeos y tomamuestras; procedimentales, manejo, análisis, 
interpretación y aplicación de la normativa para la determinación de la campaña de reconocimiento, y simulación y elaboración de una campaña para un caso real; y actitudinales, todos los enumerados en el mapa de contenidos.

- Desarrollo real: En la clase, el clima de trabajo fue intenso. La gran mayoría de los alumnos habían trabajado en casa y tenían algunas dudas. Sin embargo, muchas preguntas surgían tras la puesta en común con sus compañeros de grupo. La actuación de la profesora se centró en ayudar a los alumnos si no avanzaban en la resolución del problema. La dificultad principal fue que uno de los grupos no había trabajado en casa y sus miembros no pudieron seguir el ritmo de la clase. El tiempo previsto para la segunda sesión fue de 90 minutos, sin embargo, se tuvo que elevar hasta los 120. Otro problema fue la formación de los grupos. Muchos alumnos se quedaron sin grupo, por lo que la profesora tuvo que agruparlos. Los alumnos se interesaron por el trabajo y valoraron que se tratara de un caso real.

\section{A5. Cuestionario final}

- Tiempo previsto: 30 minutos, en la segunda sesión.

- Descripción: Con esta actividad se pretende analizar la evolución del aprendizaje de los alumnos comparando los resultados con los del cuestionario inicial mediante escaleras de aprendizaje. Para que la comparación sea real y los resultados no se vean comprometidos, a los alumnos se les realizan las mismas preguntas incluidas en el cuestionario inicial.

- Contenidos: Todos los contenidos incluidos en el mapa.

- Desarrollo real: Los alumnos resolvieron el cuestionario con más soltura que el anterior. Sin embargo, incidieron en que la actividad debería realizarse tras la entrega final de la A4. 


\section{Evaluación del aprendizaje de los estudiantes}

La evaluación del aprendizaje de los estudiantes se ha realizado mediante la propia observación, comparando sus conocimientos iniciales y finales y valorando el trabajo entregado correspondiente a la A4. En total, se encuestaron 24 alumnos. En la Figura 2 se muestra el avance que han experimentado los alumnos para cada una de las cuestiones analizadas. Además, se incluye la media de la puntuación obtenida para cada una de las preguntas según el cuestionario inicial y final.
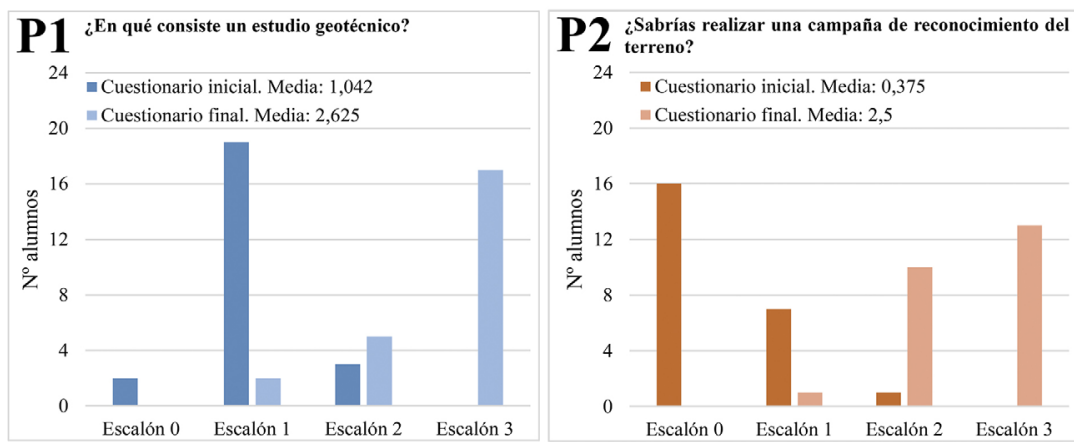

P3:Qué responsabilidad tiene el arquitecto en una campaña

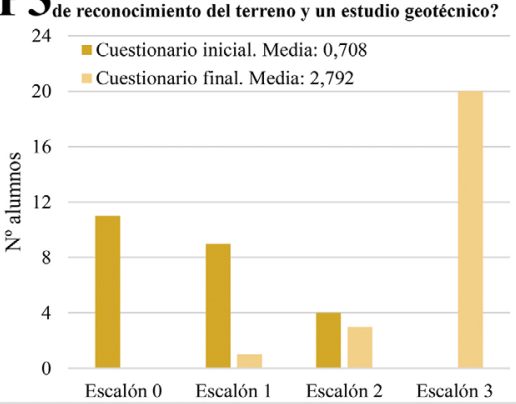

P4 iEn qué consiste el reconocimiento profundo y super-
ficial del terreno?

24 Cuestionario inicial. Media: 0,792

20 Cuestionario final. Media: 2,917

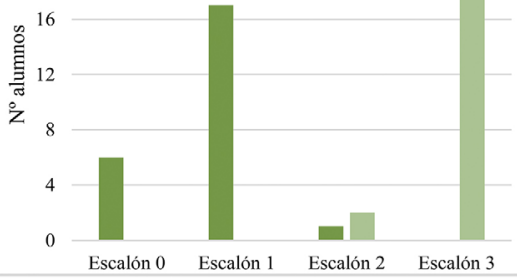

P5 ¿Qué diferencias existen entre ensayos in situ, sonDeos, tomamuestras y ensayos de laboratorio?

24 Cuestionario inicial. Media: 0,958

20 Cuestionario final. Media: 2,792

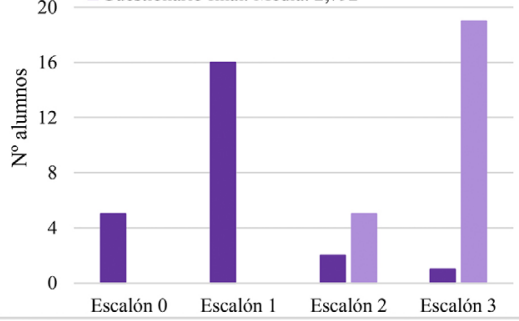

Figura 2. Escaleras de aprendizaje inicial y final en las distintas preguntas del cuestionario.

Jornadas de Formación e Innovación Docente del Profesorado I № 2 (2019) Esta obra se distribuye con la licencia Creative Commons Reconocimiento-NoComercial-SinObraDerivada 
Como puede observarse en los gráficos recogidos en la Figura 2, en general, los alumnos han mejorado notablemente su nivel de conocimiento respecto a las cuestiones preguntadas. En el primer cuestionario, la mayoría de las respuestas se han situado en el escalón 0 y en el 1. Tan solo algunas han podido ser agrupadas en el escalón 2. Se nota que los estudiantes tienen un nivel de conocimiento inicial más elevado en las preguntas 1 y 4 . Son cuestiones más sencillas que por lógica y por conocimiento general pueden responderse de forma básica sin detallar. Sin embargo, en las cuestiones más técnicas no se han obtenido datos tan positivos. En concreto, la cuestión 2 ha sido la que peores resultados ha tenido. Respecto a la pregunta 3, parece que los estudiantes conocen algo sobre la función y responsabilidad del arquitecto en este tema.

En cuanto a los resultados del cuestionario final, estos son más que satisfactorios. Sin embargo, en la cuestión 2 los estudiantes no definen bien la respuesta. Olvidan incluir que existe un procedimiento en la normativa para realizar la campaña que han tenido que comprender para resolver la A4. La mejora más sustancial se ha producido en la pregunta 3. Los alumnos han comprendido en gran medida cuál es la responsabilidad de un arquitecto en una campaña de reconocimiento del terreno y en un estudio geotécnico. Al fin y al cabo, esta es una de las cuestiones principales que se perseguían en este ciclo.

\section{Evaluación del Ciclo de Mejora en el Aula}

En líneas generales, el resultado de la aplicación del ciclo de mejora ha sido bastante satisfactorio. Los alumnos han valorado positivamente la actividad. Sin embargo, son conscientes de que es necesario mucho más tiempo para su realización. Además, requiere un mayor esfuerzo por parte de la profesora y del alumno. La profesora debe preparar la clase y los alumnos deben encontrar por sí 
mismos las herramientas teóricas necesarias para la resolución del problema.

Solo se dieron unos casos aislados en los que el CIMA no sirvió para mejorar el nivel de conocimiento y aprendizaje de los alumnos. Aun así, se puede afirmar que los alumnos asimilaron la sucesión de actividades perfectamente y que mejoraron su aprendizaje.

Para futuros ciclos de mejora, el modelo didáctico alternativo aplicado en la asignatura de Mecánica del Suelo y Cimentaciones se muestra como una mejora para el aprendizaje de los alumnos. La implementación del aprendizaje basado en problemas fomenta su capacidad de lectura, análisis e interpretación, necesarias para el desarrollo de cualquier trabajo profesional. Además, les sirve para enfrentarse a un problema real por sí solos y que en un futuro no muy lejano deberían ser capaces de resolver.

De acuerdo con García Pérez y Porlán (2017) y García Pérez (2000), los principios didácticos que han guiado la experiencia y que deben permanecer en el futuro son los siguientes:

- Para qué enseñar: asegurar la construcción de aprendizajes significativos; contribuir al desarrollo de la competencia de aprender a aprender.

- Qué enseñar: hipótesis general de construcción de conocimiento progresivo; aprendizaje a partir de mapas de contenidos por tema incluyendo contenidos conceptuales, actitudinales y procedimentales.

- Cómo enseñar: promover una participación activa; partir del nivel de desarrollo del alumnado; aprendizaje basado en problemas y secuencia coherente de actividades.

- Evaluación: centrada en la evolución del conocimiento del alumno; realizada mediante el uso de diversos instrumentos de seguimiento. 


\section{Referencias bibliográficas}

Colegio de Arquitectos de Sevilla (2010). Anejo I.2: Mapa geotécnico básico de la ciudad de Sevilla. En Protocolo de Inspección Técnica de Edificaciones.

De Alba, N. y Porlán, R. (2017). La metodología de enseñanza. En R. Porlán (Coord.), Enseñanza Universitaria. Cómo mejorarla (pp. 37-54). Madrid: Morata.

Finkel, D. (2008). Dar clase con la boca cerrada. Valencia: Publicacions de la Universitat de València.

García Pérez, F. F. (2000). Los modelos didácticos como instrumento de análisis y de intervención en la realidad educativa. Biblio 3W. Revista Bibliográfica de Geografia y Ciencias Sociales, V(207), 18 de febrero de 2000. Recuperado de http://www.ub.edu/geocrit/b3w-207.htm.

García Pérez, F. F. y Porlán, R. (2017). Los principios didácticos y el modelo didáctico personal. En R. Porlán (Coord.), Enseñanza Universitaria. Cómo mejorarla (pp. 93-101). Madrid: Morata.

Justo Moscardó, E., Delgado Trujillo, A., Vázquez Boza, M. y Branda, L. (2016). Implementation of Problem-Based Learning in Structural Engineering: A Case Study. International Journal of Engineering Education, 32(6), 2556-2568.

Ministerio de Fomento de España. (2007). Documento Básico de Seguridad Estructural Cimientos (DB-SE-C). Recuperado de https://www.codigotecnico.org/images/ stories/pdf/seguridadEstructural/DBSE-C.pdf

Rivero, A. y Porlán, R. (2017). La evaluación de la enseñanza universitaria. En R. Porlán (Coord.), Enseñanza Universitaria. Cómo mejorarla (pp. 73-92). Madrid: Morata.

Jornadas de Formación e Innovación Docente del Profesorado | № 2 (2019) Esta obra se distribuye con la licencia Creative Commons Reconocimiento-NoComercial-SinObraDerivada Internacional (CC BY-NC-ND 4.0.) 